\title{
Vitamin D serum concentration is not related to the activity of spondyloarthritis - preliminary study
}

\author{
Zofia Guła ${ }^{1}$, Anna Kopczyńska², Karolina Hańska², Maciej Słomski², Jarosław Nowakowski ${ }^{1}$, \\ Beata Kwaśny-Krochin ${ }^{1}$, Jerzy Gąsowski ${ }^{3}$, Mariusz Korkosz ${ }^{1}$ \\ ${ }^{1}$ Department of Rheumatology and Balneology, Faculty of Medicine, Jagiellonian University Medical College, Kraków, Poland \\ ${ }^{2}$ Students Scientific Group, Faculty of Medicine, Jagiellonian University Medical College, Kraków, Poland \\ ${ }^{3}$ Chair of Internal Diseases and Gerontology, Faculty of Medicine, Jagiellonian University Medical College, Kraków, Poland
}

\begin{abstract}
Objective: Vitamin D plays an important role in mineral turnover and bone remodeling and there are increasing data about its immunomodulatory potential in different rheumatologic disorders. Deficiency of vitamin D is frequent in patients with spondyloarthritis (SpA) and some data suggest its association with increased disease activity and structural damage. However, its exact role in the pathogenesis of SpA and its association with disease activity are still a matter of debate.

Material and methods: A cross-sectional study of patients diagnosed with axial spondyloarthritis (axSpA) and peripheral spondyloarthritis (perSpA) according to Assessment of Spondyloarthritis International Society classification criteria was performed. The correlation between concentration of 25-hydroxyvitamin D - 25(OH)D - and disease activity scores (Bath Ankylosing Spondylitis Disease Activity Index - BASDAI, Ankylosing Spondylitis Disease Activity Score - ASDAS), inflammatory markers (C-reactive protein - CRP, erythrocyte sedimentation rate - ESR) and clinical symptoms (arthritis, enthesitis, dactylitis) was performed.

Results: We included 40 patients with axSpA and 23 patients with perSpA. The mean concentration of 25(OH)D was $24.9 \mathrm{ng} / \mathrm{ml}$ (SD 12.49). Forty-seven (74.6\%) patients had 25(OH)D below the recommended threshold $(<30 \mathrm{ng} / \mathrm{ml})$. We found no statistically significant negative correlation between the level of 25(OH)D and disease activity of axSpA and perSpA in terms of clinical symptoms (arthritis, enthesitis, dactylitis), inflammatory markers (ESR, CRP) and disease activity scores (BASDAI, ASDAS). These results did not change after adjustment for supplementation of vitamin D and seasonal variation.

Conclusions: Our data show no correlation between the concentration of $25(\mathrm{OH}) \mathrm{D}$ in the serum and disease activity in two subgroups of SpA. However, this does not exclude the potential role of vitamin $D$ in pathogenesis of SpA. Further studies are required to evaluate the optimal range of $25(\mathrm{OH}) \mathrm{D}$ serum concentration in axSpA and perSpA patients with its possible immunomodulatory potential and influence on disease activity.
\end{abstract}

Key words: vitamin D deficiency, spondyloarthritis, disease activity.

\section{Introduction}

Deficiency of vitamin $D$ is frequent in patients with spondyloarthritis (SpA) [1, 2] and some data suggest its association with increased disease activity and structural damage $[3,4]$. Apart from the endocrine role in bone metabolism by maintaining mineral homeostasis, vitamin $D_{3}$ can modulate both innate and adaptive immune responses. Notably, vitamin D modulates immune processes inducing a shift from a pro-inflammatory T-helper 1 (Th-1), Th-17 to an anti-inflammatory Th-2 and Treg pro-

Address for correspondence:

Zofia Guła, Department of Rheumatology and Balneology, Faculty of Medicine, Jagiellonian University Medical College, 10 Śniadeckich St., 31-531 Kraków, Poland, e-mail: mcm.zofia@gmail.com

Submitted: 29.09.2018; Accepted: 30.11.2018 
file [5] and possibly influences cytokine pathways involved in SpA pathogenesis, especially IL-12/IL-23 and IL-17 [6].

Vitamin $D_{3}$ has been shown to modulate maturation and function of monocytes. As a part of the innate immune system monocytes seem to play an important role in the pathogenesis of SpA. Recently, a negative correlation between CD16 monocytes and peripheral arthritis in early SpA was observed [7]. In experimental studies vitamin D inhibited TNF and IL-1 secretion by monocytes [8]. Vitamin D up-regulated CD14 expression and down-regulated TLR2, TLR4 and TLR9 expression on human monocytes resulting in less IL-6 secretion [9]. However, this immunomodulatory effect requires a high $1,25(\mathrm{OH})_{2} \mathrm{D}_{3}$ concentration in the immune cell microenvironment [10], probably difficult to achieve under reference concentrations in blood. Likewise, serum concentration of $25(\mathrm{OH}) \mathrm{D}$ may not fully reflect the functional status of $1,25(\mathrm{OH})_{2} \mathrm{D}_{3}$ in the tissues given that proposed immune mechanisms are likely auto-/paracrine [5].

Several studies have shown dissimilar results for the relationship between serum $25(\mathrm{OH})$ D concentration and activity of SpA. Zhao et al. [3] found significantly higher Bath Ankylosing Spondylitis Disease Activity Index (BASDAI), spinal pain visual analogue scale (VAS), erythrocyte sedimentation rate (ESR) and C-reactive protein (CRP) in axial spondyloarthritis (axSpA) patients deficient in vitamin $25(\mathrm{OH}) \mathrm{D}(<30 \mathrm{nmol} / \mathrm{l})$ as compared to non-deficient patients. Also Hmamouchi et al. [4] reported higher BASDAI, Ankylosing Spondylitis Disease Activity Score (ASDAS) and radiological sacroiliitis score in early axSpA deficient for vitamin 25(OH)D (<50 nmol/l).

In a study by Erten et al. [1] vitamin D levels were inversely related with both ESR and CRP concentration in AS patients, but not in undifferentiated SpA. In this study $25(\mathrm{OH}) \mathrm{D}$ did not correlate with BASDAl in either group of patients. On the other hand, several reports did not confirm an association between vitamin D and ESR, CRP and BASDAl in axSpA [2, 11]. Also in a recent study of AS patients, Klingberg et al. [12] found no association between vitamin D deficiency $(<50 \mathrm{nmol} / \mathrm{l})$ and disease activity, osteoproliferation, BMD, vertebral fractures or bowel inflammation.

The aim of our study was to evaluate whether there would be a link between serum 25(OH)D concentration and disease activity in patients with axSpA and/or peripheral spondyloarthritis (perSpA).

\section{Material and methods}

Our study included consecutive patients with axSpA or perSpA according to Assessment of Spondyloarthritis International Society (ASAS) classification criteria. Some patients fulfilled New York criteria of ankylosing spondylitis (AS) and/or classification criteria for psoriatic arthritis (PSA). During the visit a history of supplementation of vitamin $D$ and blood samples for assessment of ESR, CRP and $25(\mathrm{OH}) \mathrm{D}$ level were taken and physical examination for presence of tender/swollen joints (TJC/SJC), enthesitis and dactylitis was performed. The range of laboratory standards for vitamin 25(OH)D serum concentration in our laboratory is $30-80 \mathrm{ng} / \mathrm{ml}$. Disease activity was assessed by BASDAI) and ASDAS.

Patients provided signed informed consent and the study protocol was approved by a local bioethics committee (no. 122.6120.44.2015).

The data management and analysis were performed with SAS version 9.2 (SAS Institute Inc., Cary, NC, USA). Summary data are presented as means and standard deviations, or, in the case of non-normally distributed variables, as medians with inter-quartile ranges. We used the Spearman correlation to check for the relationships between analyzed variables. For the comparisons of values of studied variables across categories of vitamin D concentrations we employed non-parametric tests.

\section{Results}

We included 40 patients with axSpA and 23 patients with perSpA. Overall, diagnoses included 29 patients with AS, 11 with non-radiographic axSpA, 12 with PsA, and 11 with perSpA. Patients were under 45 years, 22 (34.9\%) female and 41 (65.1\%) male. Table I presents clinical and laboratory characteristics of the study group.

The mean concentration of 25(OH)D was $24.9 \mathrm{ng} / \mathrm{ml}$ (SD 12.49) and was comparable in axSpA vs. perSpA (24 [SD 12.7] vs. 26.5 [SD 12.3], $p=0.45)$. Forty-seven (74.6\%) patients had $25(\mathrm{OH}) \mathrm{D}$ below the recommended threshold $(<30 \mathrm{ng} / \mathrm{ml})$. We found no statistically significant negative correlation between the level of $25(\mathrm{OH}) \mathrm{D}$ and disease activity of axSpA and perSpA in terms of clinical symptoms (arthritis, enthesitis, dactylitis), inflammatory markers (ESR, CRP) and disease activity scores (BASDAI, ASDAS). We found no association between level of vitamin $D$ and the intensity of therapy (nonsteroidal antiinflammatory drugs vs. conventional/biologic disease modifying drugs). We found a positive correlation between level of vitamin D and ESR in axSpA (Table II).

Spondyloarthritis subgroups analysis of patients with low $25(\mathrm{OH})$ D concentration $(\leq 20 \mathrm{ng} / \mathrm{ml})$ compared to higher 25(OH)D concentration (> $20 \mathrm{ng} / \mathrm{ml}$ ) also showed no difference in ESR (7.5 vs. 12), CRP (1.94 vs. $2.98 \mathrm{mg} /$ l), BASDAl (2.35 vs. 3.3) or ASDAS (1.55 vs. 1.91). These results did not change after adjustment for supplementation of vitamin $\mathrm{D}$ and seasonal variation. 
Table I. Clinical and laboratory characteristics of studied group

\begin{tabular}{|lccc|}
\hline Characteristic & axSpA $(n=40)$ & perSpA $(n=23)$ & $p$ \\
\hline TJC $\geq 1$ (\%) & 17.5 & 78.3 & $<0.0001$ \\
\hline SJC $\geq 1$ (\%) & 12.5 & 69.6 & $<0.0001$ \\
\hline Enthesitis $\geq 1$ (\%) & 2.5 & 43.5 & $<0.0001$ \\
\hline Dactylitis $\geq 1$ (\%) & 0 & 13 & 0.02 \\
\hline ESR median (5-95 percentile) & $8(2-46)$ & $12(5-32)$ & 0.39 \\
\hline CRP median (5-95 percentile) & $1.3(0-27.5)$ & $3(0-23)$ & 0.54 \\
\hline BASDAI median (5-95 percentile) & $2.4(0.6-7)$ & $3.8(0.4-9)$ & 0.16 \\
\hline ASDAS median (5-95 percentile) & $1.7(0.5-4.2)$ & $2.1(0.1-4.4)$ & 0.5 \\
\hline Duration of symptoms, mean $( \pm S D)$ months & $7.9(7.4)$ & $5.6(6)$ & NS \\
\hline
\end{tabular}

axSpA - axial spondyloarthritis; perSpA - peripheral spondyloarthritis; TJC - tender joints count; SJC - swollen joints count; ESR - erythrocyte sedimentation rate; CRP - C-reactive protein; BASDAI - Bath Ankylosing Spondylitis Disease Activity Index; ASDAS - Ankylosing Spondylitis Disease Activity Score; NS - not significant

Table II. Correlation between vitamin D concentration and disease activity

\begin{tabular}{|lcccc|}
\hline Characteristic & \multicolumn{2}{c}{ axSpA } & \multicolumn{2}{c|}{ perSpA } \\
\cline { 2 - 5 } & $r$ & $p$ & $r$ & $p$ \\
\hline TCJ & -0.5 & 0.75 & -0.2 & 0.94 \\
\hline SJC & 0.12 & 0.46 & 0 & 1 \\
\hline Enthesitis & -0.24 & 0.13 & -0.1 & 0.65 \\
\hline Dactylitis & - & - & -0.13 & 0.55 \\
\hline ESR & 0.45 & 0.005 & -0.02 & 0.94 \\
\hline CRP & 0.26 & 0.11 & -0.09 & 0.69 \\
\hline BASDAI & 0.14 & 0.38 & -0.38 & 0.08 \\
\hline ASDAS & 0.14 & 0.38 & -0.35 & 0.1 \\
\hline
\end{tabular}

axSpA - axial spondyloarthritis; perSpA - peripheral spondyloarthritis; TJC - tender joints count; SJC - swollen joints count; ESR - erythrocyte sedimentation rate; CRP - C-reactive protein; BASDAI - Bath Ankylosing Spondylitis Disease Activity Index; ASDAS - Ankylosing Spondylitis Disease Activity Score

\section{Discussion}

In our study we did not find a significant association between serum 25(OH)D concentration and activity of SpA. These results are in line with several previous studies that showed no direct link between serum concentration of vitamin D and ESR, CRP, BASDAI and structural damage (osteoproliferation and osteoporosis) [2, 11, 12]. However, this does not exclude the potential role of vitamin D in pathophysiology of SpA. First, the range of normal concentrations of vitamin $D$ in serum may be much lower than expected to modulate inflammatory processes. Studies showing effects of vitamin D upon immunologic cells were performed in vitro where the concentration of vitamin D was much greater than those measured in vivo [13]. Achieving such high levels of vitamin D in humans is linked with a significant risk of hypercalcemia. New agents such as selective VDR agonists, e.g. paricalcitol (19-nor-1,25-hydroxy-vitamin $D_{2}$ ), may be of interest in inhibition of the inflammatory process without disturbing calcium/phosphorus metabolism [10], but it must be confirmed in clinical trials. Second, serum concentration of $25(\mathrm{OH}) \mathrm{D}$ may not reflect the concentration of its active metabolite, $1,25(\mathrm{OH})_{2} \mathrm{D}_{3}$ in the inflammatory microenvironment. Possibly, not only serum vitamin $\mathrm{D}$ concentration, but local production of $1,25(\mathrm{OH})_{2} \mathrm{D}_{3}$ by macrophages, dendritic cells and T lymphocytes $[13,14]$ is pivotal in the auto and paracrine immunomodulatory effect.

In our analyses we found a positive correlation between level of vitamin D and ESR in axSpA. This finding is difficult to explain in the clinical context and must be confirmed by other observations.

Our results are contrary to data from other studies that reported a significant association between vitamin D and activity of SpA as well as structural damage $[1,3,4]$. However, differences in the study population and in the definition of vitamin deficiency do not allow direct comparison of these results. We also cannot exclude sig- 
nificant bias due to the geographic latitude of the study centers. The strength of our study is that our patients were young, with relatively short duration of signs and symptoms and relatively good physical function, which exclude the impact of immobilization on vitamin D status. We also performed adjustment for seasonal variation and supplementation of vitamin D, which did not show any significant differences.

\section{Conclusions}

To summarize, although achieving the recommended concentration of vitamin 25(OH)D is necessary due to its important role in bone metabolism, it still cannot be considered as a therapeutic option for inflammatory processes in SpA. It is a matter of debate what the optimal concentration of $25(\mathrm{OH}) \mathrm{D}$ in serum is, that would have immunomodulatory potential, without posing a risk of hypercalcemia. In order to elucidate the relationship between serum vitamin D deficiency and SpA activity, randomized placebo-controlled trials are required.

The authors declare no conflict of interest.

\section{References}

1. Erten S, Kucuksahin O, Sahin A, et al. Decreased Plasma Vitamin D Levels in Patients with Undifferentiated Spondyloarthritis and Ankylosing Spondylitis. Intern Med 2013; 52: 339344.

2. Mermerci Baskan B, Pekin Dogan Y, Sivas F, et al. The relation between osteoporosis and vitamin $\mathrm{D}$ levels and disease activity in ankylosing spondylitis. Rheumatol Int 2010; 30: 375-381.

3. Zhao S, Thong D, Duffield S, et al. Vitamin D Deficiency in Axial Spondyloarthritis is Associated With Higher Disease Activity. Arch Rheumatol 2017; 32: 209-215.

4. Hmamouchi I, Paternotte S, Molto A, et al. Vitamin D, disease activity and comorbidities in early spondyloarthritis. Clin Exp Rheumatol 2016; 34: 396-403.
5. Mora JR, Iwata M, von Andrian UH. Vitamin effects on the immune system. Nat Rev Immunol 2008; 8: 685-698.

6. Melis L, Elewaut D. Progress in spondylarthritis. Immunopathogenesis of spondyloarthritis: which cells drive disease? Arthritis Res Ther 2009; 11: 233.

7. Guła Z, Stec M, Rutkowska-Zapała M, et al. The absolute number of circulating nonclassical $\left(\mathrm{CD} 14^{+} \mathrm{CD} 16^{++}\right)$monocytes negatively correlates with DAS28 and swollen joint count in patients with peripheral spondyloarthritis. Pol Arch Intern Med 2017; 127: 846-853.

8. Du T, Zhou ZG, You S, et al. Regulation by 1,25-dihydroxy-vitamin D3 on altered TLRs expression and response to ligands of monocyte from autoimmune diabetes. Clin Chim Acta 2009; 402: 133-138.

9. Dickie LJ, Church LD, Coulthard LR, et al. Vitamin D3 down-regulates intracellular Toll-like receptor 9 expression and Toll-like receptor 9-induced IL-6 production in human monocytes. Rheumatology 2010; 49: 1466-1471.

10. Guillot X, Prati C, Wendling D. Vitamin D and spondyloarthritis. Expert Rev Clin Immunol 2014; 10: 1581-1589.

11. Arends S, Spoorenberg A, Bruyn GAW, et al. The relation between bone mineral density, bone turnover markers, and vitamin D status in ankylosing spondylitis patients with active disease: a cross-sectional analysis. Osteoporos Int 2011; 22: 1431-1439.

12. Klingberg E, Oleröd G, Hammarsten O, et al. The vitamin D status in ankylosing spondylitis in relation to intestinal inflammation, disease activity, and bone health: a cross-sectional study. Osteoporos Int 2016; 27: 2027-2033.

13. Hewison M, Freeman L, Hughes SV, et al. Differential Regulation of Vitamin D Receptor and Its Ligand in Human Monocyte-Derived Dendritic Cells. J Immunol 2003; 170: 5382-5390.

14. Fritsche J, Mondal K, Ehrnsperger A, et al. Regulation of 25 -hydroxyvitamin $D_{3}-1 \alpha$-hydroxylase and production of $1 \alpha$, 25-dihydroxyvitamin $\mathrm{D}_{3}$ by human dendritic cells. Clin Endocrinol (Oxf) 2003; 102: 3314-3316. 